\title{
Multi-sensor Information Fusion and its Application Analysis
}

\author{
Yan Zhang \\ Xi'an Peihua University, Xi'an, Shaanxi, 710125, China
}

Keywords: Multi-sensor; information fusion; application.

\begin{abstract}
From the last century, multi-sensor information fusion has been well developed on a global scale. This technology mainly uses computer technology to carry out automatic analysis and synthesis under the corresponding criteria by multi-sensor or multi-source information data, so as to complete the information processing work required by the required decision and estimation. The wide application of this technology has brought great benefits to the work and life and learning of modern people, and promoted the improvement of information technology in China. Based on the description of multi-sensor information fusion, this paper discusses the main algorithms of multi-sensor information fusion, and proposes the application of multi-sensor information fusion in different fields.
\end{abstract}

\section{Introduction}

In recent years, multi-sensor information fusion technology has received considerable attention both in military and civil matters. This emerging frontier research topic of information fusion is widely used in the processing of multi-sensor information. It fully utilizes the differentiated features of various types of sensors, and can achieve the corresponding goals through multiple directions. This technology has been widely used in various fields. Multi-sensor information fusion mainly refers to the fusion of data collected by different knowledge sources and sensors, so as to better understand these observed phenomena. The process of multi-sensor information fusion is quite complicated, and there are quite a lot of problems to be studied, and there are many countermeasures to solve the problem. Therefore, it is necessary to implement a comprehensive and systematic introduction to the current research situation.

\section{Overview of multi-sensor information fusion}

The so-called multi-sensor information fusion mainly refers to the use of computer technology mastered by modern people, using specific rules, automatic analysis and processing of information and data among multiple sensors, in order to achieve a complete set of information science processing process. This technology is used in the military field. Its use in the military field is mainly used to deal with detection technology. It has considerable research in several aspects such as estimation and interconnection, and can obtain more accurate identity estimation and threat estimation, etc., which can be used for military Probing work brings greater benefits. In the continuous development and evolution of society, multi-sensors have evolved into a fusion of information and data. Studies have shown that the information processing and analysis functions covered in multi-sensor systems have greatly exceeded the human brain's ability to understand information. The human brain serves as a naturalization system for understanding, analyzing and processing related information data. All the what is seen and heard will be gathered in the brain, and each visual or auditory and olfactory will be integrated and then estimated. . In this way, humans can understand everything around them. The multi-sensor information fusion system has dozens of times the ability to dispose of information and data to the human brain. The system is used in related industries and certainly has an ultra-high information processing capability. As long as the valuable resources of multi-sensors can be fully utilized, and the above sensors can be rationalized, the relevant information and data in each 
multi-sensor system can be complemented in time and space, so that it can be measured in the multi-sensor system. The object is to optimize the information processing capability of the multi-sensor itself. However, it is more worth noting that when using multi-sensor information fusion, the data in the multi-sensor may have different characteristics, sometimes even real-time data, and sometimes non-real-time data, a large number. The horizontal lines of data support or complement each other, and sometimes these data have contradictions with each other. Therefore, it is necessary to pay sufficient attention to the above problems when implementing multi-sensor information fusion.

\section{The main algorithm of multi-sensor information fusion}

The main content of multi-sensor information fusion method research is the algorithm related to data fusion. The essence of multi-sensor fusion is to process the information of multi-source uncertainty, and the uncertainty of the information can be randomized, either in the form of fuzzy pre-test information or in the form of non-test information. The mathematical tool function in multi-sensor information fusion is the most basic. It scientifically describes all the input data in a common space, and at the same time, it integrates the above data and finally outputs it. The most used tools or methods in the information fusion process are probability theory, fuzzy theory and neural networks. In addition to the above several common methods, there are some other solutions.

First, probability theory. Probability theory has a long history of development. It can successfully deal with a large number of problems related to uncertainty, and has a very rich theoretical and systematic approach. The earliest application of system fusion technology is probability theory. The phenomena it explores are completely random, that is, random variables are used to express uncertain information, and uncertain information is also processed. In a public space, the input data should be modeled according to a probability or likelihood function. Under certain prior probability conditions, the probability of each output hypothesis can be obtained according to the probability of combining Bayesian rules. In this way, the problem of uncertainty can be dealt with. Probabilistic and statistically relevant methods commonly used in this field include estimation theory, Kalman filtering, hypothesis testing, and Bayesian methods.

Second, logical reasoning. It mainly covers probabilistic reasoning, evidence reasoning, fuzzy reasoning and production rules. These are all uncertain reasoning, which can be said to be one of the most important topics in artificial intelligence exploration. The reasoning method of uncertainty is to measure the uncertain information first, and the different representations will form different uncertainty reasoning methods. Some researchers have conducted a comprehensive exploration of uncertainty reasoning, and based on this, proposed a new theory of inclusion. This theory explores not only the general principles of deterministic reasoning, but also the methodology of uncertainty. Compared with probabilistic statistics, logical reasoning has quite a number of advantages, mainly because it can overcome many problems faced by probability theory to a certain extent. At the same time, because logical description has a considerable subjective factor for the description of information, the representation and processing of information lacks objectivity. Some researchers have also conducted in-depth research on DS evidence theory. Evidence theory plays a very important role in the implementation of inference network analysis. Information specificity and certainty can be combined according to different decision-making methods. The advantages of evidence reasoning in uncertainty representation and measurement and combination are highly valued. On the basis of improving their own deficiencies, they should be combined with other methods and extended to probability ranges and fuzzy sets. This not only can be used to contact a priori information like Bayesian reasoning, but also to deal with ambiguous proof of concept as a language. Insufficient is that the results are often too high estimates, there will be new algorithms for conflict processing that is not done, and small changes in the input data will have a considerable impact on the output. Once the processing object is more compatible, its performance will also deteriorate.

Third, the fuzzy theory. This theory is based on the local theory of classification, so there are a 
large number of fuzzy classification techniques that need to be developed since the day of its creation. The membership function can embody the meaning of words, thus forming a convenient interactive interface between digital expression and symbolic expression. In the use of information fusion, it is common to model the expertise using rules that are interrelated with features. At the same time, fuzzy theory can be used to implement digital or information modeling in a strict or loose manner. Another aspect of the fuzzy theory is that it can deal with inexact description problems and adaptively merge relevant information. For the fuzzy expansion in the estimation process, it can solve the problem of information or decision conflict, and apply it to sensor fusion.

Fourth, the neural network. This network is mainly formed by a large-scale interconnected processing unit, which is based on the research results of modern neurobiology and cognitive science in information processing. The time that neural network is used in information fusion is not very long. It has the characteristics of large-scale parallel simulation processing, continuous time dynamics and network globality, and it has very strong adaptive learning ability. This can replace complex and time-consuming traditional algorithms, making the signal processing process closer to the thinking activities of modern people. Using the high-speed parallel computing function of neural network, the optimal signal processing algorithm can be realized in real time; using the distributed information storage and parallel processing of neural network, it can avoid the modeling and feature extraction in the pattern recognition mode. The process. This eliminates the adverse effects of model mismatch and unreasonable feature selection, and enables real-time recognition to improve the performance of the recognition system. Layers or nodes in a neural network can be linked to each other in a variety of forms, and a nonlinear transformation is performed on the input vector. Once the relationship between the input and output is not true, a satisfactory result can be obtained. In order to improve the acquisition rate, probability or evidence distribution data, the neural network technology and the aforementioned theory can also be combined. For example, when dealing with conflicting information and other issues, compared with the neural network method based on iterative optimization, the clustering method has lower computational complexity, but the performance is also less. Therefore, the Potts spin theory can be connected with the evidence reasoning, and the rapid neural network clustering method can be obtained, so as to properly balance the above problems.

\section{The application of multi-sensor information fusion in different fields}

First, the use in the military field. In order to safeguard national security, each country will invest huge human, financial and material resources in the military field. China naturally has a very large investment in multi-sensor information fusion, and it has also achieved very good results. Many countries, such as Britain and the United States, have researched a large number of military systems. The above systems all have quite good information fusion. The multi-sensor system developed by the UK covers information fusion systems such as artillery intelligence, maneuvering and control. The most advanced aspect of distributed data fusion developed by the United Kingdom is the rational use of distributed technology in data fusion technology for scientific combinatorial optimization. China's information fusion technology is centralized data, and all information or data is completed in the same level. The disadvantage of centralized systems is that once the central node is damaged or attacked, it will cause damage to the multi-sensor information fusion system, but distributed information fusion will not produce this situation. All runs are performed on each node. As a result, even if a node is attacked or destroyed, it will not cause a system-wide crash. The UK company that developed the system has experimented with the system and succeeded. This experiment involves eight different nodes, and the form of networking is used in the above nodes. This system can implement the autonomous layout of the nodes. Once the enemy's trace is found in the positioning radar, the autonomous drone can receive the above information in the first time and fly to the relevant area for viewing. Surveillance radar can be tracked on the ground, and even if the tracking target does not appear in the line of sight, virtual tracking or alerting can be performed at all times. This advantage is hard to match with other systems. In this way, the accuracy and security of the information data collected in the sensor system is improved. 
Second, the use in the civil field. In view of the continuous development of China's economy and society, multi-sensor information fusion technology has developed into the civilian industry, and has achieved excellent results in agriculture and other industries. For example, it can be applied to technologies such as intelligent transportation, industrial robot fault diagnosis and car unmanned driving. In the car unmanned operation, this technology uses the control system to develop the driverless function of the car. The system is equipped with positioning system, inertial sensor and digital map. It can control the specific driving direction of the car in real time, and it can automatically detect the specific conditions of the ground section. At the same time, the system can also detect front road conditions and obstacles, so that driverless cars have greater safety and stability. After the development of driverless cars, many people doubted the safety performance, but it turned out that the driverless car after the introduction of multi-sensor information fusion technology has greater security.

\section{Conclusions}

In summary, in view of the rapid development of artificial intelligence technology in recent years, information fusion technology has also developed toward the trend of intelligence and systematization. The future research direction of this technology will be based on multi-sensors operating in a dynamic environment, and the fusion method will become a research hotspot. It is sure to be a very important evolutionary path to closely link various computational intelligence technologies such as fuzzy logic, rough set theory and wavelet transform. In this way, information processing tools will certainly show very important value, and artificial intelligence and neural network methods will inevitably continue to be a research hotspot. Therefore, based on this research, we should continue to strengthen the exploration of multi-sensor information fusion and its application, in order to further promote the development of China's information technology field.

\section{Acknowledgement}

This research was financially supported by 1. Research project of Shaanxi Provincial Department of Education special research program, Wireless sensor network positioning algorithm based on pulse ultra-wideband technology, project number: 17JK1059; 2. Xi'an Peihua College, school-level project, "Research on UAV visual tracking technology", No. (PHKT18104); 3. Xi'an Peihua College, School-level project, "Research on Dynamic Migration Algorithm of Open Flow Switch Based on Attractor Selection in Cloud Environment", No. (PHKT18062).

\section{References}

[1] Yang Shengguang. Discussion on Multi-sensor Information Fusion Technology and Its Application Prospect in Agricultural Engineering, Agricultural Technology Service, 2015(6).

[2] Wang Junna, Lei Jing. Overview of Multi-sensor Information Fusion and Its Application, Information Recording Materials, 2016(5).

[3] Wang Hongxin. Application of multi-sensor information fusion theory in relative navigation of drones, Journal of National University of Defense Technology, 2017(5).

[4] Zhou Hao. Application of Multi-sensor Information Fusion Technology in Intelligent Driving System, Technology \& Market, 2018(4). 